\title{
Effectiveness of duloxetine in treatment of painful chemotherapy-induced peripheral neuropathy: a systematic review
}

\author{
Wael Ibrahim, MD, and Ahmed AM Abdelhafeez, $\mathrm{MD}^{\mathrm{b}}$
}

\author{
Departments of a Neurology and ${ }^{b}$ Clinical Oncology, Kasralainy Hospital, Faculty of Medicine, Cairo University, Egypt
}

Chemotherapy-induced peripheral neuropathy (CIPN) is a serious side effect that can be dose limiting and affect patient quality of life. To date, the therapeutic options for CIPN are limited. We performed a systematic literature search of the PubMed and Scopus databases to assess the effectiveness of duloxetine in the treatment of pain in patients with CIPN. The search included randomized controlled trials, nonrandomized controlled trials, retrospective studies, and single-arm studies of duloxetine in treatment of CIPN. A descriptive analysis of the studies was performed. The PubMed database online search identified 41 publications, and a second database search through Scopus identified 29 publications. A total of 10 full-text articles were assessed for eligibility, with 5 articles excluded. Altogether, the included studies reported 431 patients with painful CIPN. An improvement in pain scores was the primary and/or secondary endpoint in the included studies. Pain was assessed by 6 different scores. Comparator drugs were used in 4 studies in our review. The comparator drug was placebo in 1 study only, and the remaining 3 studies used other antineurotoxicity therapy. The chemotherapeutic agents used in the studies were the following: paclitaxel $(52.9 \%)$, oxaliplatin (39.7\%), R-CHOP (rituximab, doxorubicin, vincristine, and cyclophosphamide; 3.30\%), combined bortezomib-dexamethasone $(1.89 \%)$, FOLFOX (folinic acid, fluorouracil, and oxaliplatin; $1.18 \%)$, and other taxanes (0.94\%). From the descriptive analyses, and from the available data of relatively small sample sized studies, it can be concluded that despite the above limitations, duloxetine remains a useful therapeutic option for pain in CIPN patients, regardless of the type of chemotherapeutic agent used.

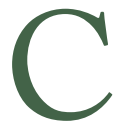
hemotherapy-induced peripheral neuropathy (CIPN) is a serious side effect that can be dose limiting and affect patient quality of life for prolonged time, ${ }^{1}$ with an overall incidence of about $38 \%$ in patients who are treated with multiple chemotherapeutic agents. ${ }^{2} \mathrm{CIPN}$ has various clinical presentations - affecting the motor, sensory, and autonomic nerves - but the most common manifestations are numbness, tingling, and burning pain affecting the upper and lower extremities (the stocking-and-glove distribution)..$^{3-5}$ It can also lead to numerous negative effects on activities of daily living, functioning, ${ }^{6}$ leisure activities, dressing, household and work activities, going barefoot or wearing shoes, and driving. The incidence of CIPN is variable, depending on many factors such as type of chemotherapy, total dose, dose per cycle, infusion duration, and comorbidities as diabetes mellitus. ${ }^{5-7}$

The most common antineoplastic agents causing peripheral neuropathy are oxaliplatin, cisplatin, taxanes, Vinca alkaloids, bortezomib, and thalidomide. ${ }^{3,8,9}$

Different components of the nervous system are targets of various chemotherapeutic agents, from dorsal root ganglion (DRG) cells to the distal axon.
The DRG is the most vulnerable to neurotoxicity because it is less protected by the nervous system blood barrier, hence the predominance of sensory symptoms in CIPN. ${ }^{10}$ The pathogenesis of CIPN is not fully understood, and it is most probably multifaceted and not always related to the antineoplastic mechanism. Findings from experimental studies have shown an accumulation of chemotherapeutic compounds in the cell bodies of the DRG, resulting in decreased cellular metabolism and axoplasmic transport. Another proposed mechanism is the induction of apoptosis in sensory neuron of the posterior spinal ganglion after binding to DNA strands. ${ }^{7,11}$

Opioids had been used for managing pain in patients with cancer, but their addictive side effects limit use in the treatment of chronic pain, ${ }^{12}$ so several drugs called coanalgesics have been introduced as a treatment for CIPN, including antidepressants (tricyclic antidepressants, serotonin [5HT], and norepinephrine $[\mathrm{NE}]$ reuptake inhibitors), anticonvulsants (carbamazepine, and gabapentin), topical lidocaine patch, and topical gel. ${ }^{13}$ Duloxetine has been shown to be effective as a treatment option for

Accepted for publication November 20, 2018. Correspondence: Wael Ibrahim, MD; dr.wael_ezzał@hotmail.com. Disclosures: The authors report no disclosures or conflicts of interest. JCSO 2018;16(6):e243-e249. (02018 Frontline Medical Communications. doi: https://doi.org/10.12788/jicso.0436 
painful diabetic neuropathy, ${ }^{14-16}$ but there is a lack of data on its effectiveness in patients with CIPN..$^{17,18}$ To date, the therapeutic options for CIPN remain limited. ${ }^{12,13,19}$

The imbalance of 5HT and NE in the pain inhibitory pathways may contribute to the peripheral neuropathic pain. ${ }^{20}$ Duloxetine hydrochloride is a $5 \mathrm{HT}-\mathrm{NE}$ reuptake inhibitor used to treat depression and generalized anxiety disorder. ${ }^{21}$ Duloxetine effect in decreasing pain transmission through increasing synaptic concentrations of $\mathrm{NE}$ and $5 \mathrm{HT}$, which results in blocking input signals to the dorsal horn neurons in the spinal cord. ${ }^{12}$

\section{Methods}

We followed the Preferred Reporting Items for Systematic Reviews and Meta-Analyses (PRISMA statement) guidelines during the preparation of this systematic review. ${ }^{22}$

\section{Inclusion criteria}

Trial or study type. Articles publishing findings from randomized controlled trials, nonrandomized controlled trials, retrospective studies, and single-arm studies of duloxetine in the treatment of CIPN were included in our review.

Intervention. The intervention was duloxetine with all doses, either administered alone or with other antineuropathic drugs.

Comparator. The comparator was placebo (control group) or other antineuropathic drugs or no control group.

Population. The population included cancer patients with painful CIPN.

Outcome. At least one of the following outcomes was used for pain assessment: visual analog scale (VAS) score; Brief Pain Inventory-Short Form (BPI-SF), neuropathic pain score using National Cancer Institute's Common Toxicity Criteria for Adverse Events, version 3 or version 4 (NCICTCAE v3.0, v4.0), or Functional Assessment of Cancer Therapy-Taxane (FACT-Tax) Scales.

\section{Exclusion criteria}

Studies in a non-English language, animal studies, studies whose full-text article was not available, and thesis and conference papers were not included.

\section{Objective and study design}

The objective of this systematic review was to systematically assess the effectiveness of duloxetine in the treatment of pain in patients with CIPN.

\section{Information sources and search}

Medical electronic databases. PubMed and Scopus, from inception to January 2018, were searched using the follow- ing search queries: (((duloxetine) AND chemotherapy induced peripheral neuropathy)) OR (((chemotherapy) AND (neuropathic pain OR peripheral neuropathy))) AND duloxetine).

Selection of studies. The authors selected eligible studies. The screening of search results was performed in the following 2 steps:

- Screen title and abstracts against the selection criteria. Articles that were unclear from their title or abstract were reviewed against the selection criteria through the full text.

- Retrieve and screen full-text articles of eligible abstracts for eligibility to systematic review.

\section{Data extraction}

Two authors extracted the following data independently: sample size, mean age, chemotherapeutic drug, duloxetine dosage, and outcomes for pain assessment using at least one score from VAS, BPI-SF, neuropathic pain score using the NCI-CTCAE v3.0 and v4.0, or FACT-Tax, and other secondary outcomes. The data was exported from the online forms as a Microsoft Excel sheet.

\section{Statistical analysis}

We calculated the mean age and associated standard deviations (SDs) for all patients by using the pooled mean and pooled SD equation, according to Cochrane handbook of systematic reviews of interventions 5.1.0 (updated March 2011). ${ }^{23}$ When data are expressed as median and interquartile range, we used Hozo and colleagues' BMC Research Methodology equation to calculate or estimate the mean and SD. ${ }^{24}$

Data are expressed as means with SD (unless stated otherwise); statistical results were considered significant when the $P$-value was less than .05. Data analysis was performed using the SPSS Statistical Package, version 23 (IBM Corp., Armonk, NY).

\section{Synthesis of data and analysis}

Because of heterogeneity and low sample size of studies, no statistically justified analyses could be performed on the provided data. Instead, a descriptive analysis of published studies was performed.

\section{Summary measures}

The search strings, the list of relevant reviews, the data coding, and the quality criteria that were used can be requested from the corresponding author.

\section{Results}

\section{Selection of articles}

The systematic literature search and subsequent selection are summarized in a flow diagram (Figure). The PubMed database online search identified 41 publications, and a 
second database search through Scopus identified 29 publications. After 27 duplicate publications were removed, a total of 43 publications were screened for title and abstract. All articles with animal instead of human patients, review articles as well as articles not written in the English language were excluded ( $\mathrm{n}$ $=33$ articles). A total of 10 full-text articles were assessed for eligibility, with 5 being excluded for the following reasons: full text not available $(n=1)$, review article $(n=2)$, secondary analysis $(n=1)$, and primary outcome not met $(n=1)$.

\section{Study characteristics}

Characteristics of the included studies and patient outcome are summarized in Table 1 and Table 2. A total of 5 studies from 2012 through 2017 were included in the descriptive analysis and systematic review. In all, 4 trials were prospective studies, and 1 trial was retrospective; among all trials, 2 studies were single arm and 3 were placebocontrolled and/or crossover.

\section{Baseline characteristics of included studies}

There were 431 participants in the total 5 studies included in this systematic review. The number of patients per study ranged from 25 to 231. Patients were mostly older, with mean sample ages ranging from 47.9 to 63 years, and the pooled mean age for all participants in the total 5 studies was 57.7 years.

In all included studies, duloxetine was given in varying doses of $20 \mathrm{mg}, 30 \mathrm{mg}$, $40 \mathrm{mg}$, or $60 \mathrm{mg}$. Also, different therapeutic regimens of duloxetine were used, including placebo control or crossover with vitamin B12; 80\% of the studies used escalation of doses over time (only 1 trial used fixed doses for each group of patients in the study). Escalation of duloxetine by doubling the dose was done in all 4 studies, with duloxetine doses of $30 \mathrm{mg}$ and $60 \mathrm{mg}$ used in $75 \%$ of those studies ( 3 out of 4 studies).

Comparator drug was used in 4 studies ( 1 was single arm) in our review analysis. The comparator drug was placebo in 1 study only, and the remaining 3 studies used other antineurotoxicity or antineuropathic pain therapy, mainly vitamin B12 (as only comparator in 1 study), fish oil, prega- balin, selective $5 \mathrm{HT}$ reuptake inhibitors, and nonsteroidal anti-inflammatory agents.

Regarding CIPN, the chemotherapeutic agents used in the studies were as follows (after exclusion of 11 patients who never received treatment in 1 study): 224 patients (52.9\%) were on paclitaxel, $168(39.7 \%)$ on oxaliplatin, $14(3.30 \%)$ on R-CHOP, 8 (1.89\%) on combined bortezomib-dexamethasone, 5 (1.18\%) on FOLFOX, and 4 (0.94\%) on other taxanes.

Improvement in pain scores was the primary and/or secondary endpoint in the included studies (Table 2). Pain was assessed by 6 different scores, including the VAS, BPI-SF, neuropathic pain score using NCI-CTCAE v3.0 and v4.0, and FACT-Tax, with all reported once except the VAS score, which was reported in 2 studies. Only 1 study, by Yang and colleagues, ${ }^{25}$ measured pain by 2 scores (the VAS and NCI- 
TABLE 1 Baseline characteristics of included studies

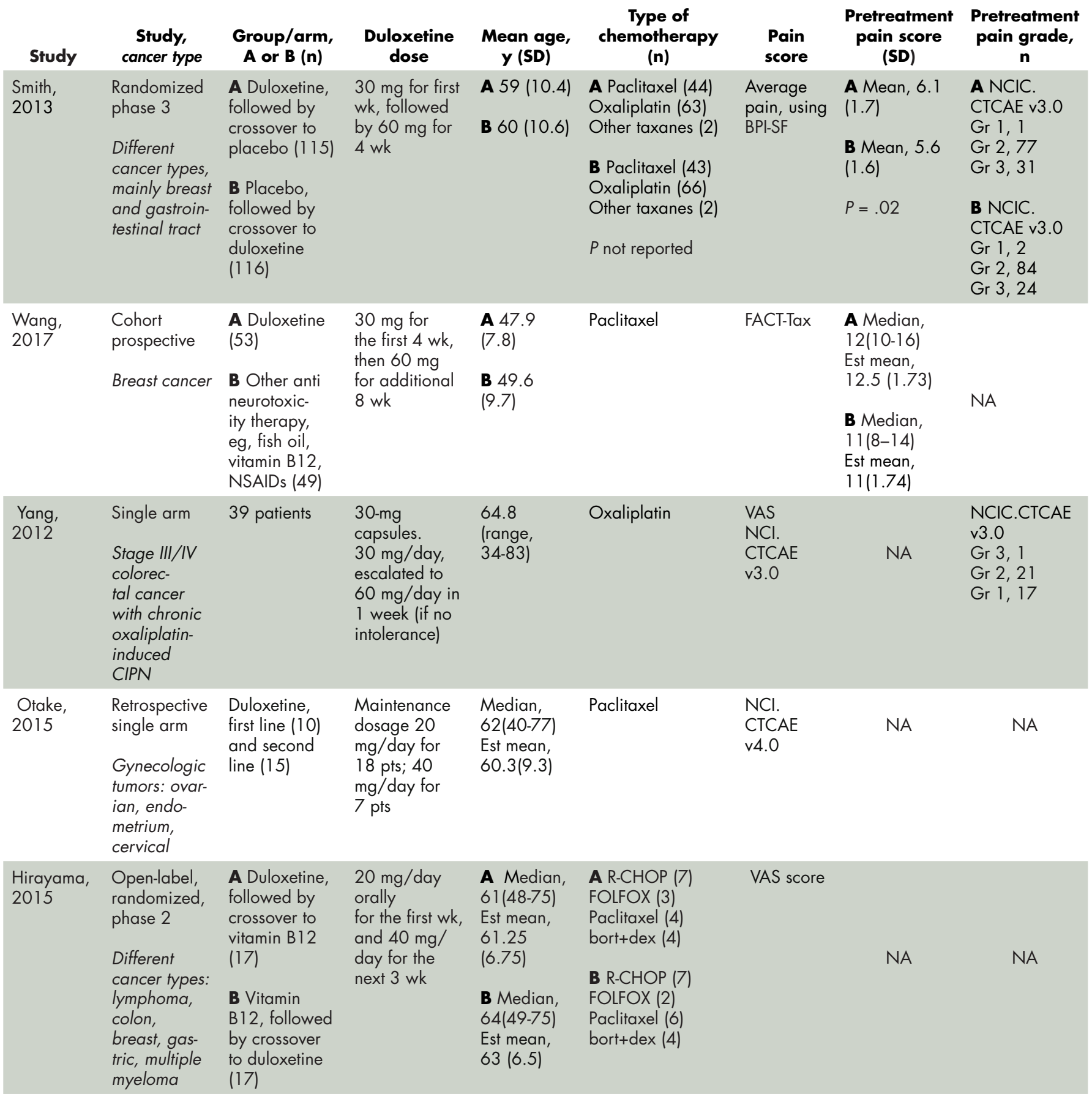

bort+dex, bortezomib plus dexamethasone; BPI-SF, Brief Pain Inventory-Short Form; CIPN, chemotherapy-induced peripheral neuropathy; Est, estimated; FACT-Tax, Functional Assessment of Cancer Therapy-Taxane; FOLFOX, folinic acid, fluorouracil, and oxaliplatin; Gr, grade; NA, not applicable; NCI.CTCAE v3.0, National Cancer Institute's Common Toxicity Criteria for Adverse Events, version 3 or 4; NSAIDs, nonsteroidal anti-inflammatory drugs; pts, patients; R-CHOP, rituximab, doxorubicin, vincristine, and cyclophosphamide; VAS, visual analog scale

CTCAE v3.0), with the rest of the studies assessing pain by just 1 of the aforementioned scores. The pretreatment pain score was reported in only 2 studies, by Smith and colleagues and Wang and colleagues, using BPI-SF and FACT-Tax scores, respectively, with total respective mean scores of 5.8 $(\mathrm{SD}, 1.7)$ and $11.77(\mathrm{SD}, 1.73) .^{17,26}$

Secondary endpoints were related mainly to pain score, drug adverse effect, and assessment of quality of 
TABLE 2 Outcome summary of included studies

Study

Smith, 2013

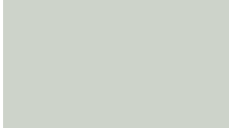

Otake, 2015

Res

Yang, 2012

Primary endpoint

Mean difference in the average pain score was $0.73195 \%$ $\mathrm{Cl}, 0.26-1.20)$ from start to end of the initial treatment period (wk 1 to wk 5).

Mean change score in Arm A, $1.06(95 \% \mathrm{Cl}, 0.72-1.40)$ Mean change score in Arm B, $0.34(95 \% \mathrm{Cl}, 0.01-0.66)$ $(P=.003)$

Wang, 2017 Decrease in the severity of paclitaxel-induced CIPN (OR, $5.426 ; 95 \% \mathrm{Cl}, 1.898-15.514 ; P=.002)$.

The median (25th-75th percentiles) decrease of FACT-Tax pain score in the duloxetine and control groups was $4(2-6)$ and $1(0-4)$, respectively $(P=.005)$.

\section{Subjective response based on VAS scores was seen in 19} pts $(63.3 \%)$.

9 pts $(47.4 \%)$ showed a simultaneous objective grade improvement ( $\mathrm{Gr} 3$ to $2, \mathrm{n}=1 ; \mathrm{Gr} 2$ to $1, \mathrm{n}=8)$, and 10 pts $(52.6 \%)$ maintained a stable grade $(\mathrm{Gr} 2, \mathrm{n}=4 ; \mathrm{Gr}$, $\mathrm{n}=6$ ), according to $\mathrm{NCl}$.CTCAE v3.0.

Response (improvement) seen in $14 / 25$ pts (56\%).
$20 \mathrm{mg} /$ day, $O R=1$
$40 \mathrm{mg} /$ day, $O R=0.64(95 \% \mathrm{Cl}, 0.078-5.2)$

Hirayama, 2015 Hazard ratio for $\geq 30 \%$ and $\geq 50 \%$ reduction in numbness for duloxetine vs vitamin B12 were 0.25 and 0.40 , respectively.

Hazard ratio for $\geq 30 \%$ and $\geq 50 \%$ reduction in pain

for duloxetine vs vitamin B12 were 0.28 and 0.25 , respectively.

\section{Secondary endpoint}

Mean difference in the FACT/GOG-Ntx was $1.58195 \% \mathrm{Cl}$, $0.15-3.00 ; P=.03$ ).

Mean difference of BPI-SF interference score was 4.40 (95\% Cl, 0.93-7.88; $P=.01$ ).

Nonneuropathic adverse events that are attributed to chemotherapy were mild and similar in both groups.

No significant differences were observed in the incidence of paclitaxel-induced CIPN.

9 pts $(28.1 \%)$ discontinued duloxetine because of intolerable adverse events, including dizziness/giddiness/nausea $(n=4)$, restlessness/insomnia $(n=2)$, somnolence $(n=2)$, and urinary hesitancy $(n=1)$.

Adverse events were very mild and usually well tolerated, including somnolence $(n=3,12 \%)$, giddiness $(n=3$, $12 \%)$, nausea $(n=1,4 \%)$, constipation $(n=1,4 \%)$, dysgeusia/distortion of taste $(\mathrm{n}=1,4 \%)$.

All adverse events were $\mathrm{Gr} 1$, including fatigue $(n=6$, $17.6 \%)$, nausea $(n=3,8.8 \%)$, somnolence $(n=2,5.9 \%)$, and insomnia $(\mathrm{n}=2,5.9 \%)$.

BPI-SF, Brief Pain Inventory-Short Form; Cl, confidence interval; CIPN, chemotherapy-induced peripheral neuropathy; FACT-Tax, Functional Assessment of Cancer Therapy-Taxane; GOG-Ntx, Gynecologic Oncology Group-Neurotoxicity; Gr, grade; NCI.CTCAE v3.0, National Cancer Institute's Common Toxicity Criteria for Adverse Events, version 3 or 4; OR, odds ratio; pts, patients; VAS, visual analog scale

life (Table 2). In the study by Yang and colleagues, ${ }^{25} 9$ patients $(28.1 \%)$ discontinued duloxetine because of intolerable adverse events, with dizziness or giddiness as the most common cause $(44.4 \%$ of patients who discontinued treatment). Studies by Otake and colleagues ${ }^{12}$ and Hirayama and colleagues ${ }^{2}$ reported duloxetine adverse events that were very mild and usually well tolerated in collectively 22 patients, with fatigue $(n=6)$ and somnolence $(n=5)$ as the most reported adverse effects. Wang and colleagues ${ }^{17}$ reported nonneuropathic adverse events that were attributed to chemotherapy and were mild and similar in both study groups.

\section{Discussion}

To our knowledge, this is the first systematic review to discuss the effectiveness of duloxetine specifically in treatment of pain in CIPN. The administration of chemotherapeutic agents such as paclitaxel, cisplatin, oxaliplatin, and vincristine was accompanied by CIPN. The currently available treatment options for CIPN are limited. To date, no drug has been approved specifically for treatment of pain in CIPN. ${ }^{12}$

In our review, we included cancer patients with CIPN and associated pain. Several previous studies ${ }^{8,27,28}$ discussed tingling and numbness as a common adverse effect in CIPN, and usually about $20 \%$ to $42 \%$ of patients develop chronic pain.

Six different pain assessment scores were reported in the total 5 studies in our review, with VAS and NCI-CTCAE scores reported in more than 1 study. This reflects the major challenges facing the assessment of CIPN, as various scales and tools are available for pain assessment but without a standardized approach for CIPN that can be precisely implemented. ${ }^{8}$ Several other challenges regarding pain scores were observed, with Smith and colleagues as the only authors to report both pretreatment pain score and grade, while the rest of the studies failed to report either pain score or grade, or even both.

Another difficulty observed in our review was the variability in study participants in both population size and 
type of cancer treated. The population size in largest study included in our review was 231 patients and the smallest was 25 patients; collectively, there were only 431 patients in the included studies. Although the type of primary cancer varied in between studies, gynecologic malignancies comprised most cases (215 patients), followed by gastrointestinal tumors, and few cases of hematologic and genitourinary malignancies were reported. Similar results were observed by Geber and colleagues in their large study screening pain in cancer patients, in which gynecologic malignancies were diagnosed in 28 patients out of 61 with CIPN, representing the highest percentage (45.9\%) of malignancy type in that study. ${ }^{26}$

In the study by Otake and colleagues ${ }^{12}$ examining duloxetine for CIPN in patients with gynecologic cancer, the authors concluded that duloxetine dosage either $20 \mathrm{mg} /$ day or $40 \mathrm{mg} /$ day was not associated with the effectiveness of duloxetine treatment by either univariate or multivariate analysis. Previous authors have provided an explanation for the difference in duloxetine response among CIPN patients and attributed those differences to the underlying pain mechanisms. ${ }^{14,29}$ In other words, pain in those patients is both peripheral nociceptive and central neuropathic, and it is likely to be caused by mixed mechanisms.

Another variation observed among CIPN patients in our review was the chemotherapeutic agents used. That was noted by Smith and colleagues, ${ }^{26}$ who reported that patients with cancer who received platinum therapies (oxaliplatin) experienced more benefit from duloxetine in terms of pain improvement than those who received taxanes $(P=.13)$. We found no other published studies on the response to duloxetine among different chemotherapeutic agents used. However, 2 studies of duloxetine response in patients with other pain-related disorders (painful diabetic peripheral neuropathy and fibromyalgia) showed significant improvement in pain symptoms compared with placebo. In a study of pain in chemotherapy-induced neuropathy $(\mathrm{CIN})$ by Geber and colleagues, ${ }^{29}$ the preexisting pain medication was not reported, but the authors concluded that treatment for CIN-related neuropathic pain differs from that for nonneuropathic (ie, musculoskeletal) pain, with the former being treated mainly with pharmacotherapy and the latter with physiotherapy and behavioral exercises. They asserted that different pain patterns could help flag neuropathic or musculoskeletal pain so that the selected treatments would be more specific. Differences in pain improvement related to duloxetine may be attributed to the underlying pain mechanism, and whether it is mixed or centrally or peripherally related was also discussed by Geber and colleagues. ${ }^{29}$

In the study by Geber and colleagues, the chemotherapeutic protocols comprised a combination of chemotherapeutic agents so that the symptoms and signs of CIPN could not be attributed to a single agent. ${ }^{29}$ By contrast, all the studies included in our review used a chemotherapeutic protocol with single agent so that specific symptoms and signs of CIPN could be attributed to an individual chemotherapeutic agent.

Findings from studies on the effect of duloxetine in treatment of pain in diabetic peripheral neuropathy have shown that duloxetine at a dose of $60 \mathrm{mg} /$ day effectively improves pain in $43 \%$ to $68 \%$ of patients. ${ }^{15,16,30}$ Similarly, in our review, the study by Yang and colleagues ${ }^{25}$ showed a $63 \%$ subjective reduction in pain severity by VAS score in CIPN patients but lower improvement of $47.4 \%$ by NCI-CTCAE v3.0; this can be attributed to the simplistic 4 -grade rating scale of the latter.

During our analysis of studies, we noticed that no diagnostic criteria were implemented for diagnosis or inclusion of CIPN patients in any of the included studies, and this represents a major challenge in any analysis of studies with neuropathic pain patients. In 2016, Finnerup and colleagues updated the previous 2008 grading system for diagnosis of neuropathic pain, which is intended to determine the level of certainty with which the pain in question is neuropathic. ${ }^{31}$ The system defines the diagnostic certainty into 3 levels: Possible, Probable, and Definite. Although the number of studies used the grading system during the inclusion of neuropathic pain patients increased from 5\% in 2009 to $30 \%$ in 2014 , still more than two-thirds of studies do not use a standardized system for diagnosis and/or inclusion of neuropathic pain in patients.

\section{Strength and limitations}

The first strength of this review is that it identifies gaps in our current knowledge about duloxetine in the treatment of pain in cancer patients with CIPN. Second, we collected all available articles from inception until January 2018. Third, this review can serve as a model for future studies investigating the effectiveness of duloxetine in treatment of CIPN.

There are also limitations to this review that should be discussed. First, the studies vary greatly in samples, methodologies, and outcomes measured. Second, the diagnostic criteria for CIPN and the pain assessment tools vary among the studies. Third, there is also variability in the duloxetine doses and administration regimens among the studies, and some articles did not report the precise outcome in pain scores. Furthermore, the articles reviewed included retrospective, single-arm, or nonrandomized controlled studies with relatively small numbers of participants.

To improve the results, more placebo-controlled or head-to-head trials (with other agents used in treatment of CIPN) with large sample sizes would be needed.

\section{Conclusions}

Our purpose was to describe the effectiveness of duloxetine in improving pain scores among CIPN patients, but because 
of heterogeneity, the low sample size of available studies, and lack of high-quality evidence, we were only able to perform a descriptive analysis of published studies. From the descriptive analyses and from the available data of relatively small sample sized studies, it can be concluded that despite the aforementioned limitations, duloxetine remains a useful therapeutic option for pain in CIPN patients, regardless of the type of chemotherapeutic agent used.

\section{Acknowledgments}

That authors express their sincere gratitude to Nahla A Merghany and Sarah M Abd Elfadel for helping them retrieve all the relevant articles for this review.

\section{References}

1. Windebank AJ, Grisold W. Chemotherapy-induced neuropathy. J Peripher Nerv Syst. 2008;13(1):27-46.

2. Hirayama Y, Ishitani K, Sato Y, et al. Effect of duloxetine in Japanese patients with chemotherapy-induced peripheral neuropathy: a pilot randomized trial. Int J Clin Oncol. 2015;20(5):866-871.

3. Stubblefield MD, McNeely ML, Alfano CM, Mayer DK. A prospective surveillance model for physical rehabilitation of women with breast cancer: chemotherapy-induced peripheral neuropathy. Cancer. 2012;118(suppl 8):2250-2260.

4. Park SB, Goldstein D, Krishnan AV, et al. Chemotherapy-induced peripheral neurotoxicity: a critical analysis. CA Cancer J Clin. 2013;63(6):419-437.

5. Argyriou AA, Kyritsis AP, Makatsoris T, Kalofonos HP. Chemotherapy-induced peripheral neuropathy in adults: a comprehensive update of the literature. Cancer Manag Res. 2014;6(1):135-147.

6. Bakitas MA. Background noise: the experience of chemotherapyinduced peripheral neuropathy. Nurs Res. 2007;56(5):323-331.

7. Miltenburg NC, Boogerd W. Chemotherapy-induced neuropathy: a comprehensive survey. Cancer Treat Rev. 2014;40(7):872-882.

8. Hausheer FH, Schilsky RL, Bain S, Berghorn EJ, Lieberman F. Diagnosis, management, and evaluation of chemotherapy-induced peripheral neuropathy. Semin Oncol. 2006;33(1):15-49.

9. Park SB, Krishnan AV, Lin CS, Goldstein D, Friedlander M, Kiernan MC. Mechanisms underlying chemotherapy-induced neurotoxicity and the potential for neuroprotective strategies. Curr Med Chem. 2008;15(29):3081-3094.

10. Caponero R, Montarroyos ES, Tahamtani SMM. Postchemotherapy neuropathy. Rev Dor. Sao Paulo. 2016;17(suppl 1):S56-S58.

11. Velasco R, Bruna J. Chemotherapy-induced peripheral neuropathy: an unresolved issue. Neurologia. 2010;25(2):116-131.

12. Otake A, Yoshino K, Ueda Y, et al. Usefulness of duloxetine for paclitaxel-induced peripheral neuropathy treatment in gynecological cancer patients. Anticancer Res. 2015;35(1):359-363.

13. Hershman DL, Lacchetti C, Dworkin RH, et al. Prevention and management of chemotherapy-induced peripheral neuropathy in survivors of adult cancers: American Society of Clinical Oncology clinical practice guideline. J Clin Oncol. 2014;32(18):1941-1967.

14. Smith EM, Pang H, Ye C, et al. Predictors of duloxetine response in patients with oxaliplatin-induced painful chemotherapy-induced peripheral neuropathy (CIPN): a secondary analysis of randomised controlled trial - CALGB/alliance 170601 [published online November 25, 2015]. Eur J Cancer Care (Eng1). 2017;26(2). doi:10.1111/ecc.12421

15. Goldstein DJ, Lu Y, Detke MJ, Lee TC, Iyengar S. Duloxetine vs placebo in patients with painful diabetic neuropathy. Pain. 2005;116(1-2):109-118.

16. Raskin J, Pritchett YL, Wang F, et al. A double-blind, random-

ized multicenter trial comparing duloxetine with placebo in the management of diabetic peripheral neuropathic pain. Pain Med. 2005;6(5):346-356.

17. Wang J, Li Q, Xu B, Zhang T, Chen S, Luo Y. Efficacy and safety of duloxetine in Chinese breast cancer patients with paclitaxel-induced peripheral neuropathy. Chin J Cancer Res. 2017;29(5):411-418.

18. Irving G, Tanenberg RJ, Raskin J, Risser RC, Malcolm S. Comparative safety and tolerability of duloxetine vs pregabalin vs duloxetine plus gabapentin in patients with diabetic peripheral neuropathic pain. Int J Clin Pract. 2014;68(9):1130-1140.

19. Esin E, Yalcin S. Neuropathic cancer pain: what we are dealing with? How to manage it? Onco Targets Ther. 2014;7:599-618.

20. Suzuki R, Rygh LJ, Dickenson AH. Bad news from the brain: descending 5-HT pathways that control spinal pain processing. Trends Pharmacol Sci. 2004;25(12):613-617.

21. Mancini M, Perna G, Rossi A, Petralia A. Use of duloxetine in patients with an anxiety disorder, or with comorbid anxiety and major depressive disorder: a review of the literature. Expert Opin Pharmacother. 2010;11(7):1167-1181.

22. Moher D, Liberati A, Tetzlaff J, Altman DG; PRISMA Group. Preferred reporting items for systematic reviews and meta-analyses: the PRISMA statement. Ann Intern Med. 2009;151(4):264-269.

23. Higgins JPT, Green S, eds. Cochrane handbook for systematic reviews of interventions. Version 5.1.0. http://handbook-5-1. cochrane.org/. Updated March 2011. Accessed November 19, 2018.

24. Hozo SP, Djulbegovic B, Hozo I. Estimating the mean and variance from the median, range, and the size of a sample. BMC Med Res Methodol. 2005;5(1):13.

25. Yang YH, Lin JK, Chen WS, et al. Duloxetine improves oxaliplatininduced neuropathy in patients with colorectal cancer: an open-label pilot study. Support Care Cancer. 2012;20(7):1491-1497.

26. Smith EM, Pang H, Cirrincione C, et al. Effect of duloxetine on pain, function, and quality of life among patients with chemotherapy-induced painful peripheral neuropathy: a randomized clinical trial. JAMA. 2013;309(13):1359-1367.

27. Dworkin RH. An overview of neuropathic pain: syndromes, symptoms, signs, and several mechanisms. Clin J Pain. 2002;18(6):343-349.

28. Cavenagh J, Good P, Ravenscroft P. Neuropathic pain: are we out of the woods yet? Intern Med J. 2006;36(4):251-255.

29. Geber C, Breimhorst M, Burbach B, et al. Pain in chemotherapy-induced neuropathy-more than neuropathic? Pain. 2013;154(12):2877-2887.

30. Wernicke JF, Pritchett YL, D'Souza DN, et al. A randomized controlled trial of duloxetine in diabetic peripheral neuropathic pain. Neurology. 2006;67(8):1411-1420.

31. Finnerup NB, Haroutounian S, Kamerman P, et al. Neuropathic pain: an updated grading system for research and clinical practice. 2016;157(8):1599-1606. 